\title{
Caractérisation de la macroporosité d'un sol de verger par analyse d'image
}

\author{
V Hallaire ${ }^{{ }^{*}}$, JP Cointepas 2 \\ 1 INRA Science du sol, 65 rue de Saint-Brieuc, 35042 Rennes; \\ 2 ENITAH, 2 rue Le Nôtre, 49005 Angers, France
}

(Reçu le 11 mai 1992; accepté le 16 octobre 1992)

\begin{abstract}
Résumé - L'analyse d'images est utilisée pour décrire la macroporosité d'un sol sous plantation de pommiers. À partir d'échantillons naturels de sols imprégnés dans une résine de polyester, la méthode fait appel à une technique de seuillage localisé permettant de distinguer la macroporosité de la matrice de sol. Les macropores individualisés sont ensuite caractérisés par 3 paramètres permettant d'estimer leur taille, leur forme et leur orientation. L'analyse porale de 5 niveaux du sol étudié met en évidence des différences que les mesures physiques de porosimétrie n'avaient pas fait apparaître : le profil montre une évolution des caractéristiques macroporales, apparaissant clairement sur les histogrammes construits selon les paramètres morphologiques. Les conséquences sur la pénétration des racines et sur les conditions de transfert de l'eau sont discutées. On montre ainsi l'intérêt d'une analyse morphologique incluant taille, forme et orientation des pores pour l'étude du comportement agronomique d'un sol
\end{abstract}

analyse d'image / porosité / verger / morphologie mathématique / indice de forme

Summary - Image analysis of the macroporosity of an orchard soil. In order to study the macroporosity of a soil of an apple orchard, image analysis was applied to soil samples impregnated with a polyester resin. The method is based on local thresholding which allows macropores and soil matrix to be distinguished. Three parameters were used for pore identification: size, shape and orientation. The porosity analysis of 5 levels in the soil allowed differences to be distinguished which were not found with physical measurements. Changes in pore characteristics were clearly distinguishable on histograms of the soil profile. Consequences of the penetration of roots and water in the soil have been discussed. Size, shape and orientation of pores could be used to predict the agronomic behaviour of the soil.

image analysis / porosity / orchard / mathématical morphology / shape index

\section{INTRODUCTION}

De nombreux travaux ont pu montrer l'influence majeure des pores les plus grossiers sur le fonctionnement hydrique des sols, et spécialement sur leurs propriétés de transferts (Bouma et al, 1977; Germann et Beven, 1981; Smettem et Collis-George, 1985; Douglas, 1986). Or, l'étude des relations entre l'organisation des matériaux et leur état hydrique a surtout permis une meilleure connaissance de leur microstructure (Croney et Coleman, 1954; Tessier, 1984; Ben Rhaïem et al, 1987), alors que la “macroporosité» souffre encore d'une absence de définition précise, due notamment à la difficulté de mettre en œuvre sur de larges champs d'analyse des méthodes d'observation mieux adaptées à des échelles plus fines : cette difficulté apparaît plus nettement encore pour les sols argileux où la diversité en taille et en forme des macropores nécessite des approches méthodologiques spécifiques aux matériaux gonflants (Reeve et Hall, 1978; Lawrence et al, 1979; Newman et Thomasson, 1979; Hallaire, 1988; Scott et al, 1988).

Si les méthodes physiques de porosimétrie permettent une mesure volumique de la phase porale, la prise en compte de la forme des pores

\footnotetext{
* Correspondance et tirés à part
} 
exige l'emploi d'outils morphologiques, ce qui a amené l'analyse d'image à connaître un important développement dans l'étude de la porosité des sols (Jongerius et al, 1972; Bullock et Thomasson, 1979; Ringrose-Voase, 1987; Warner et al, 1989).

Dans cette étude, l'analyse d'image a été utilisée pour étudier la macroporosité d'un sol sous verger et mettre en évidence ses variations entre horizons. Après avoir mis en œuvre une méthode de seuillage adaptée au matériau analysé, cette étude propose une description des macropores sur la base de 3 critères morphologiques : la taille, la forme et l'orientation des pores. Les résultats de cette analyse quantitative et qualitative de la macroporosité seront discutés en relation avec l'utilisation agronomique du sol.

\section{MATÉRIEL ET MÉTHODES}

\section{Le sol}

Le sol analysé est situé à la Morinière (Indre-et-Loire, France), dans le domaine expérimental du centre technique interprofessionnel des fruits et légumes, au centre d'un verger de pommiers âgés de 4 ans.

Le sol est développé sur des formations crayeuses turonniennes. II présente la succession d'horizons suivante :

- 0-30 cm : horizon brun; texture limono-sableuse; structure polyédrique grossière; pores tubulaires nombreux; racines de pommiers très nombreuses, principalement horizontales; transition nette;

- 30-70 $\mathrm{cm}$ : horizon brun jaunâtre à nombreuses taches d'hydromorphie grises; texture argilolimoneuse; structure polyédrique fine bien développée au sommet de l'horizon, moins nette à la base; pores tubulaires nombreux; quelques racines et radicelles de pommiers; transition diffuse;

- 70-130 cm : horizon jaune-brun à nombreuses taches d'hydromorphie grises; texture argileuse; structure prismatique fine; quelques pores tubulaires et nombreuses fissures fines décelables même en hiver racines absentes; transition graduelle;

- > $130 \mathrm{~cm}$ : horizon gris clair à nombreuses taches brunes; texture argilo-sableuse; structure massive; porosité non décelable, racines absentes.

Sur le tableau I figurent les caractéristiques granulométriques de ces horizons, ainsi que leur densité apparente sèche mesurée au densitomètre à membrane lors de la prospection pédologique (Lorgeoux, 1981). Le caractère illuvial marqué dès $30 \mathrm{~cm}$ en fait un sol lessivé hydromorphe tronqué, présentant sous un horizon superficiel Ap des horizons d'accumulation d'argile (B1g jusqu'à $70 \mathrm{~cm}$, B2tg jusqu'à $130 \mathrm{~cm}$ ), puis un horizon $\mathrm{Cg}$ d'altération issu des formations crayeuses.

\section{Échantillonnage}

Cinq niveaux d'étude ont été choisis de façon à caractériser des volumes pédologiques distincts tant par leur texture que par leur structure :

- au ccur de l'horizon de surface Ap, entre 15 et $25 \mathrm{~cm}$ de profondeur; cette zone d'étude sera dénommée 20;

- au cœur de I'horizon argilo-limoneux B1g, où la structure polyédrique est bien développée, entre 40 et $50 \mathrm{~cm}$ de profondeur; cette zone d'étude sera dénommée 45;

- à la base du même horizon B1g, où la structure polyédrique est moins bien développée, entre 60 et $70 \mathrm{~cm}$ de profondeur; cette zone d'étude sera dénommée 65 ;

- dans I'horizon argileux prismatique B2tg, entre 75 et $85 \mathrm{~cm}$ de profondeur, au cœur des prismes; cette zone d'étude sera dénommée $80 p$;

- à la même profondeur, mais à la périphérie des prismes, dans la partie incluant les fissures; cette zone d'étude sera dénommée $80 f$.

Des échantillons de sol non remaniés (hauteur $15 \mathrm{~cm}$, largeur $10 \mathrm{~cm}$, épaisseur $4-8 \mathrm{~cm}$ ) ont été prélevés en fin d'hiver à ces profondeurs dans une fosse située à l'interrang, à $1 \mathrm{~m}$ des plus proches pommiers. $\mathrm{Au}$ laboratoire, les échantillons ont subi un échange eau/acétone en phase liquide, puis ont été imprégnés par une résine de polyester (Crystic) contenant un pigment fluorescent (Uvitex) (Murphy et al, 1977). Après imprégnation, la surface des échantillons a été polie. Ces surfaces sont observées par réflexion sous éclairage ultra-violet.

Tableau I. Caractéristiques granulométriques du sol étudié, et densité apparente sèche des horizons.

\begin{tabular}{|c|c|c|c|c|c|c|}
\hline \multirow[t]{2}{*}{ Profondeur } & \multicolumn{5}{|c|}{ Fractions granulométriques (\%) } & \multirow{2}{*}{$\begin{array}{c}\text { Densité } \\
\text { apparente }\end{array}$} \\
\hline & Argile & Limon fin & Limon grossier & Sable fin & Sable grossier & \\
\hline $0-30 \mathrm{~cm}$ & 14 & 18 & 28 & 10 & 26 & 1,73 \\
\hline $30-70 \mathrm{~cm}$ & 35 & 21 & 36 & 5 & 15 & 1,46 \\
\hline $70-130 \mathrm{~cm}$ & 44 & 13 & 11 & 5 & 24 & 1,60 \\
\hline$>130 \mathrm{~cm}$ & 32 & 10 & 10 & 12 & 34 & \\
\hline
\end{tabular}


La figure 1 présente des coupes verticales de sol aux profondeurs $20 \mathrm{~cm}, 45 \mathrm{~cm}, 65 \mathrm{~cm}$ et $80 \mathrm{~cm}$; à $80 \mathrm{~cm}$, l'image présentée recouvre les 2 zones d'étude $80 f$ et $80 p$. L'analyse est effectuée sur des coupes verticales afin de pouvoir prendre en compte l'orientation des pores, mais l'observation et la mesure des pores tubulaires sur de telles coupes peuvent être affectées si leur section n'est pas perpendiculaire au plan de coupe; l'importance de l'éllipticité de la section en biais d'un pore cylindrique sur une coupe verticale sera discutée lors de l'établissement d'un indice de forme visant à distinguer les pores tubulaires des pores fissuraux.

\section{Analyse d'image}

L'analyse d'image est effectuée sur un mini-ordinateur Bull SPS7/75 doté du périphérique TITN Colorix pour
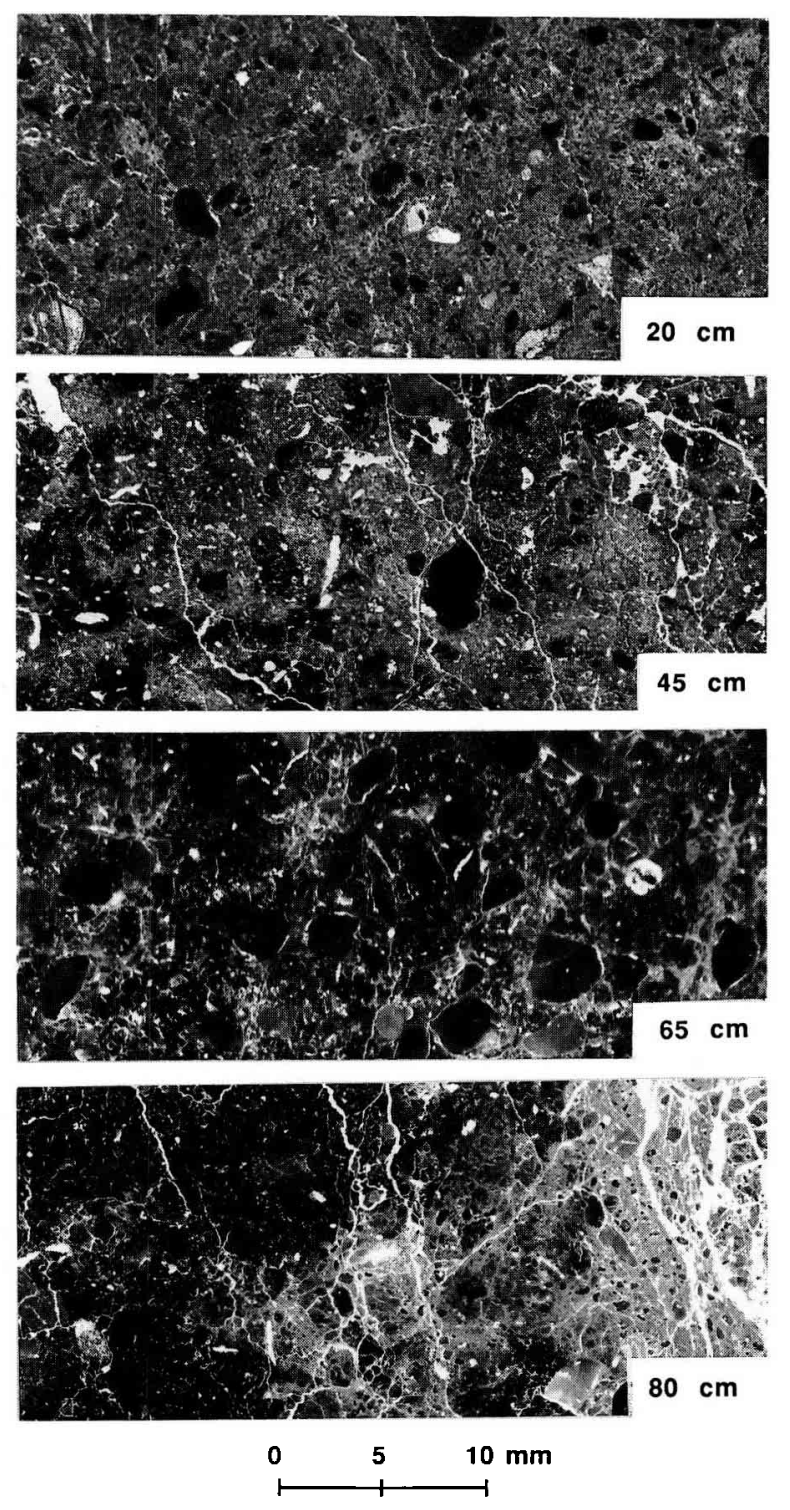

Fig 1. Coupes de sol aux profondeurs étudiées $(20 \mathrm{~cm}, 45 \mathrm{~cm}$, $65 \mathrm{~cm}$ et $80 \mathrm{~cm}$ ). Sous éclairage ultra-violet, les pores apparaissent en clair sur fond sombre. la gestion en mémoire et l'affichage sur écran des images digitalisées, et équipé du logiciel Noesis Visilog pour le traitement proprement dit des images.

L'acquisition des images est effectuée directement au moyen d'une caméra vidéo placée au-dessus des échantillons éclairés en lumière ultra-violette, sans intermédiaire d'un microscope, de façon à exclure de l'analyse la microporosité. Les images acquises sont des fenêtres carrées de $23 \mathrm{~mm}$ de côté. Pour chacune des 5 zones d'étude, 5 images ont été saisies. Les images ont été digitalisées en 512 × 512 pixels, selon une trame hexagonale, et numérisées sur 256 niveaux de gris. Chaque pixel représente ainsi une longueur de $45 \mu \mathrm{m}$, soit une surface d'environ 2000 $\mu \mathrm{m}^{2}$. La taille du pixel correspond à notre limite inférieure de résolution, choisie de façon à ne prendre en compte que la macroporosité : ne seront retenus dans l'analyse que les pores dont la taille, dans quelque direction que ce soit, est supérieure à $45 \mu \mathrm{m}$.

L'acquisition des images ayant été effectuée sous éclairage ultra-violet, les pores apparaissent en clair sur fond sombre. Mais la faiblesse du signal à détecter (faible différence de niveaux de gris entre les pores de la phase solide), et sa variation au sein même de l'image, rendent difficiles la détection automatique des pores et la binarisation des images en niveaux de gris : le plasma est constitué de plages sombres et de plages claires. Ces dernières peuvent être dues, soit à la réduction du fer, et se situer généralement autour des macropores, soit à une microporosité plus importante, occasionnant une fluorescence de masse et faisant apparaître ces zones plus claires : la différence de luminosité du fond matriciel est particulièrement visible, sur la figure 1, entre le niveau 20 et le niveau 65 . Bien qu'intentionnellement exclue de l'analyse par le choix de la résolution du pixel, la microporosité rend de ce fait difficile l'utilisation d'un seuil unique de niveaux de gris pour la détection des macropores.

Pour résoudre cette difficulté sans devoir mettre en $œ u v r e$ d'autres techniques d'acquisition (microscopie électronique à électrons rétrodiffusés), nous utiliserons une technique de seuillage localisé, c'est-à-dire tenant compte de l'environnement des objets à détecter. En utilisant les principes de la morphologie mathématique (Serra, 1982), nous adopterons la "transformation en chapeau haut-de-forme"; cette transformation comprendra les opérations suivantes :

- «ouverture" de l'image initiale. On obtient une telle "ouverture» par succession de 2 opérations de morphologie mathématique : l'érosion, qui agit sur la fonction «niveaux de gris» en assombrissant les pics clairs, et la dilatation, qui rehausse la fonction «niveaux de gris". Ces opérations sont effectuées au moyen d'un ensemble hexagonal de pixels, appelé «élément structurant", prenant successivement toutes les positions de l'image analysée; la taille choisie ici pour l'élément structurant (12 pixels) tient compte de la taille des pores à détecter et de l'échelle d'analyse. L'image résultant de cette ouverture, ou «image ouverte», représente le niveau de gris basal de la matrice solide; 
- soustraction, pixel à pixel, de l'image initiale par l'image ouverte. Le résultat de cette soustraction est une image en niveaux de gris sur laquelle les pores apparaissent de façon contrastée, quelle que soit l'intensité locale du plasma environnant;

- seuillage de cette image. Le seuil choisi (niveaux de gris supérieurs à 35) correspond au minimum de fréquence des niveaux de gris sur l'image précédente.

Sur l'image binaire ainsi réalisée, les particules connexes, c'est-à-dire les macropores, sont ensuite individualisées. Le nombre de pores reconnus dans le plan de l'image ne peut évidemment pas être assimilé au nombre de pores tel qu'on pourrait l'appréhender dans un espace tridimensionnel; contrairement à ce que permettent d'autres études nécessitant l'emploi de coupes sériées et/ou d'une modélisation morphologique de la porosité (Sullivan et Koppi, 1987; Mackie-Dawson et al, 1988; Hallaire, 1989), nous ne tenterons donc pas ici de confronter nos données surfaciques à des données volumiques.

\section{Paramétrisation des caractéristiques porales}

La quantification des caractéristiques porales se fera au moyen de 3 paramètres : la taille des pores, un indice de forme, et un indice d'orientation.

\section{Taille des pores}

L'importance de la macroporosité sera estimée de 2 façons :

- la macroporosité totale d'une image sera estimée par l'aire occupée par les pores sur l'image binaire, exprimée en pixels. Ramenée à la surface de l'image (512 x 512 pixels), cette valeur sera dénommée "macroporosité surfacique»;

- la taille de chacun des pores individualisés sera estimée par son aire individuelle, mieux apte en l'occurrence à rendre compte de cette grandeur que le diamètre des pores, utilisé lorsque la porosité est essentiellement tubulaire (Edwards et al, 1988).

\section{Forme des pores}

La classification des pores suivant leur forme a pour but de distinguer les pores tubulaires des pores fissuraux; en 2 dimensions, ces pores se distinguent par la forme de leur section, arrondie pour les uns, allongée pour les autres.

Parmi les paramètres topologiques habituellement utilisés, l'indice d'allongement (Coster et Chermant, 1985) rend bien compte de ce critère :

$$
\text { allongement }=\frac{(\text { périmètre })^{2}}{4 \pi \times \text { aire }}
$$

Cet indice est d'autant plus grand que le pore est allongé, et prend la valeur minimale 1 pour un pore rond. Malgré les réserves qu'impose son emploi dans un espace digitalisé, l'indice d'allongement s'avère utile pour classer des particules suivant leur forme (Bui et al, 1989).

On peut par ailleurs quantifier le risque d'erreur due à l'ellipticité de la section en biais d'un pore cylindrique observé sur une coupe verticale : l'indice d'allongement ne dépasse 5 que lorsque l'excentricité de l'ellipse est supérieure à 13 , c'est-à-dire lorsque l'angle entre le plan de la coupe et l'axe du pore est supérieur à $85^{\circ}$. Le risque de confusion entre un pore tubulaire vu de biais et un pore fissural, dont l'indice d'allongement peut atteindre 100 , est donc négligeable puisqu'i suppose que le plan de coupe soit tangentiel au pore.

\section{Orientation des pores}

L'orientation est un paramètre descriptif essentiel des pores allongés (Bui et Mermut, 1989) : elle peut rendre compte du fonctionnement hydrique des pores fissuraux, et plus généralement de l'arrangement structural des agrégats. Sur une section verticale où les pores ont été individualisés, l'estimation de ce paramètre a donné lieu à la mise au point de différents indices d'orientation (Murphy et al, 1977; Low et al, 1982; Pagliai et al, 1983; Daniel et al, 1987; Launeau et al, 1990).

L'orientation peut être estimée à partir des moments d'ordre 2 (selon les axes $X$ et $Y$ de l'image) du pore au sein de l'image (Noesis, 1991) :

$$
\theta=1 / 2 \operatorname{Arctg}\left(\frac{2 \cdot \operatorname{Mom} X Y}{\operatorname{Mom} X^{2}-\operatorname{Mom} Y^{2}}\right)
$$

Il apparaît cependant que cet indice doit être corrigé en fonction des diamètres de Féret horizontal (Feret $X$ ) et vertical (FeretY) du pore : ces diamètres de Féret, qui mesurent la taille du pore en projection selon les deux axes $X$ et $Y$, permettent de construire le rectangle circonscrit au pore et de déterminer si le grand axe de ce rectangle est horizontal ou vertical. Aussi proposons-nous l'emploi de l'indice d'orientation suivant :

$$
\text { ou } \begin{array}{ll}
\text { orientation }=-\theta & \text { si Feret } Y \leq \text { Feret } X \\
\text { orientation }=-\theta+90 & \text { si Ferret } Y>\text { Feret } X
\end{array}
$$

Cet indice corrigé donne un angle compris entre $0^{\circ}$ et $180^{\circ}$; il prend la valeur $0^{\circ}$ ou $180^{\circ}$ pour une ligne horizontale, et la valeur $90^{\circ}$ pour une ligne verticale.

\section{RÉSULTATS ET DISCUSSION}

Le tableau II présente, pour les 25 images analysées, le nombre de macropores reconnus dans 
Tableau II. Nombre de pores, aire totale des pores et aire individuelle moyenne des pores sur les 25 images analysées. Moyenne et écart type par niveau d'étude.

\section{Image Aire totale Aire individuelle Nombre (Pixels) (\%) (Pixels) $\left(10^{-3} . \mathrm{mm}^{2}\right)$}

\begin{tabular}{|c|c|c|c|c|c|}
\hline 20.1 & 654 & 18224 & 6,9 & 27,9 & 55,8 \\
\hline 20.2 & 612 & 17291 & 6,6 & 28,3 & 56,6 \\
\hline 20.3 & 529 & 15336 & 5,8 & 29,0 & 58,1 \\
\hline 20.4 & 633 & 18627 & 7,1 & 29,4 & 58,7 \\
\hline 20.5 & 617 & 16043 & 6,1 & 26,0 & 52,0 \\
\hline Moyenne & 609 & 17104 & 6,5 & 28,1 & 56,2 \\
\hline Écart type & 48 & 1402 & 0,5 & 1,3 & 2,6 \\
\hline 45.1 & 649 & 29220 & 11,1 & 45,0 & 90,0 \\
\hline 45.2 & 583 & 31797 & 12,1 & 54,5 & 109,1 \\
\hline 45.3 & 587 & 30098 & 11,5 & 51,3 & 102,5 \\
\hline 45.4 & 635 & 28058 & 10,7 & 44,2 & 88,4 \\
\hline 45.5 & 619 & 29873 & 11,4 & 48,3 & 96,6 \\
\hline Moyenne & 614 & 29805 & 11,4 & 48,7 & 97,4 \\
\hline Écart type & 29 & 1367 & 0,5 & 4,3 & 8,5 \\
\hline 65.1 & 368 & 16366 & 6,2 & 44,5 & 89,1 \\
\hline 65.2 & 408 & 16783 & 6,4 & 41,1 & 82,3 \\
\hline 65.3 & 301 & 17351 & 6,6 & 57,6 & 115,1 \\
\hline 65.4 & 276 & 16728 & 6,4 & 60,6 & 121,2 \\
\hline 65.5 & 293 & 17242 & 6,6 & 58,8 & 117,7 \\
\hline Moyenne & 329 & 16894 & 6,4 & 52,5 & 105,0 \\
\hline Écart type & 56 & 403 & 0,2 & 9,0 & 17,9 \\
\hline $80 f .1$ & 448 & 47875 & 18,3 & 106,9 & 213,7 \\
\hline $80 f .2$ & 916 & 43488 & 16,6 & 47,5 & 95,1 \\
\hline $80 f .3$ & 651 & 46111 & 17,6 & 70,8 & 141,6 \\
\hline $80 f .4$ & 629 & 49520 & 18,9 & 78,7 & 157,4 \\
\hline $80 f .5$ & 742 & 44147 & 16,8 & 59,5 & 119,0 \\
\hline Moyenne & 677 & 46228 & 17,6 & 72,7 & 145,4 \\
\hline Écart type & 171 & 2520 & 1,0 & 22,5 & 45,0 \\
\hline $80 p .1$ & 281 & 19559 & 7,5 & 69,6 & 139,2 \\
\hline 80p.2 & 249 & 18552 & 7,1 & 74,5 & 149,0 \\
\hline 80p.3 & 311 & 20823 & 7,9 & 67,0 & 133,9 \\
\hline $80 p .4$ & 251 & 17821 & 6,8 & 71,0 & 142,0 \\
\hline 80p.5 & 289 & 19176 & 7,3 & 66,4 & 132,8 \\
\hline Moyenne & 276 & 19186 & 7,3 & 69,7 & 139,4 \\
\hline Écart type & 26 & 1127 & 0,4 & 3,3 & 6,6 \\
\hline
\end{tabular}

l'espace bidimensionnel, leur aire totale (exprimée en pixels et en \% par rapport à la surface de l'image), et leur aire individuelle moyenne (exprimée en pixels et en $\mathrm{mm}^{2}$ ).

Les résultats montrent une faible variabilité entre les 5 images de chaque zone d'étude. Nous considérerons donc que 5 images apparaissent suffisantes pour analyser chacune de ces zones, les moyennes obtenues sur ses 5 images pouvant être considérées comme représentatives de ses caractéristiques macroporales.

\section{Analyses de la taille des pores}

Les résultats d'aire individuelle moyenne, présentés sur le tableau II, montrent que la taille moyenne des macropores varie d'un facteur 2,5 entre le niveau $20\left(0,056 \mathrm{~mm}^{2}\right)$ et les niveaux $80 f$ et $80 p\left(0,140 \mathrm{~mm}^{2}\right)$, les niveaux 45 et 65 présentant des valeurs intermédiaires. On constate par ailleurs qu'il n'y a pas de relation entre la macroporosité surfacique et la taille moyenne des pores : les niveaux 65 et $80 p$, où la porosité est faible, ont des pores en moyenne plus grossiers (plus de $0,1 \mathrm{~mm}^{2}$ ) que le niveau 45 où la porosité est beaucoup plus importante $(11,4 \%)$.

Les histogrammes de taille des pores pour les 5 niveaux étudiés (fig 2) permettent d'affiner cette analyse. L'ordonnée est exprimée en pourcentage de l'aire porale par rapport à l'aire de l'image. Les classes de taille, en abscisse, sont déterminées suivant une échelle logarithmique; à titre indicatif, pour des pores ronds, une valeur de 1 en abscisse correspondrait à un diamètre équivalent de $80 \mu \mathrm{m}$, une valeur de 6 à un diamètre de $1 \mathrm{~mm}$, un valeur de 9 à un diamètre de $5 \mathrm{~mm}$.

Le niveau $80 f$ se démarque nettement des autres niveaux, avec la présence de pores de très grande taille. Les histogrammes des autres niveaux sont tous unimodaux, mais montrent des différences qualitatives et quantitatives : les niveaux 45,65 et $80 p$ ne diffèrent que par l'intensité des pics, plus élevée en 45 qu'en 65 et $80 \mathrm{p}$; la taille moyenne des pores est donc identique dans ces 3 niveaux, mais leur nombre est plus élevé au niveau 45. Par contre I'histogramme du niveau 20 se distingue des précédents par un décalage vers la gauche : les pores sont sensiblement plus petits dans l'horizon superficiel que dans les horizons sous-jacents.

Ces informations complètent utilement les mesures physiques. La densimétrie mettait en évidence une compacité plus importante dans I'horizon de surface que dans l'horizon sousjacent; les données morphologiques montrent que la macroporosité est affectée par cette augmentation de compacité, (taille moyenne des macropores plus faible en 20 qu'en 45). Au sein de l'horizon $30-70 \mathrm{~cm}$, on a pu mettre en évi- 


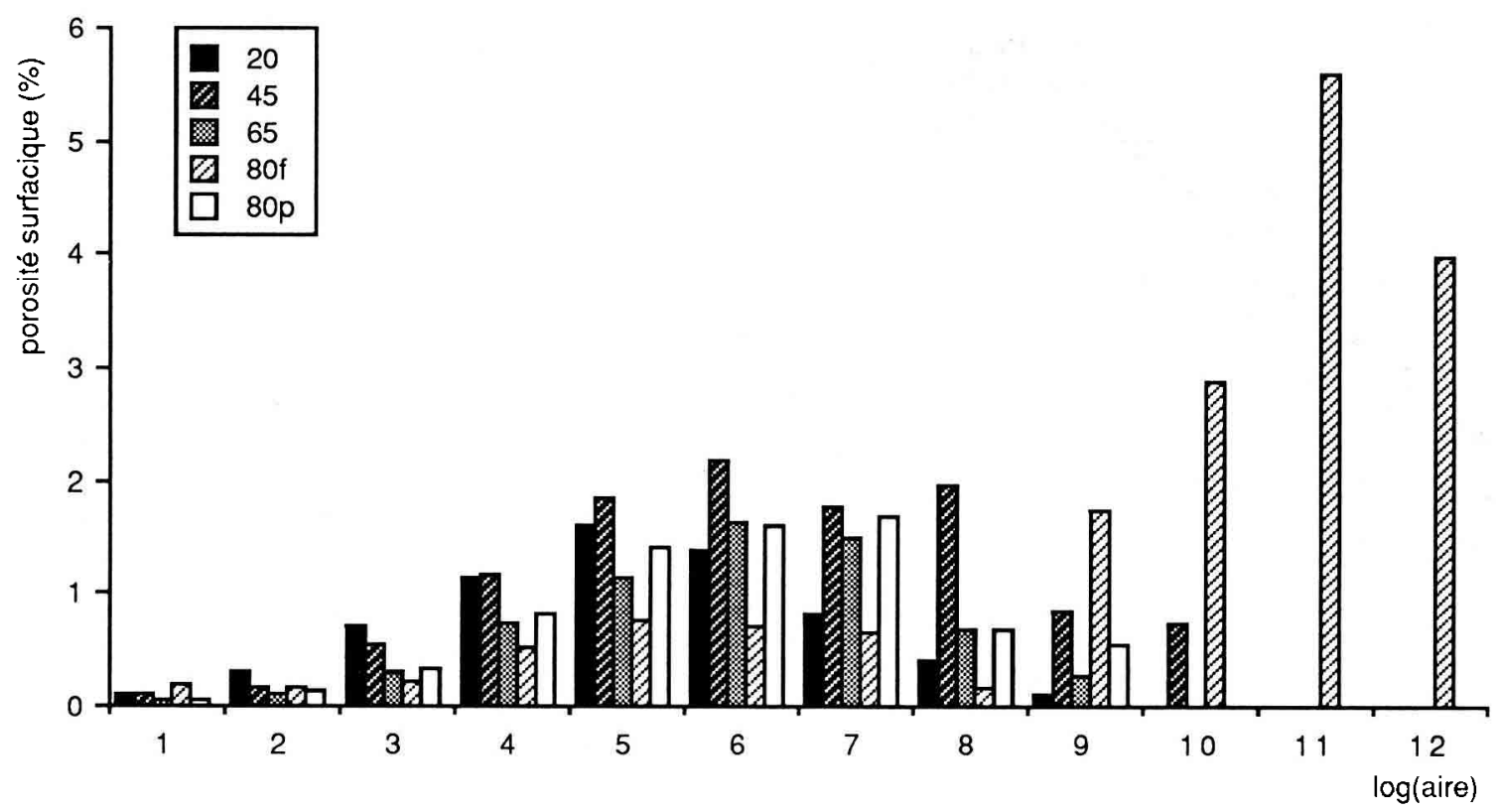

Fig 2. Histogrammes de la taille des pores pour les 5 niveaux analysés. Les classes de taille sont définies suivant le logarithme népérien de l'aire des pores (en pixels).

dence un gradient de macroporosité non pris en compte par l'analyse physique : le nombre de macropores diminue de moitié entre le haut et le bas de I'horizon. Enfin, pour l'horizon profond $70-130 \mathrm{~cm}$, nos résultats montrent d'importantes différences, en taille et en nombre de macropores, entre le cœur des primes et leur périphérie, là où l'analyse densimétrique intégrait l'ensemble de l'horizon. II apparaît ainsi que la caractérisation de la porosité d'un sol ne peut pas être uniquement effectuée par une mesure globale, mais exige une analyse individuelle des pores : seule celle-ci peut mettre en évidence une différence de taille des pores entre des niveaux de porosité semblable ( 20 et 65 ), et par contre, une similitude de taille des pores entre des niveaux de porosité différente $(45,65$ et 80p).

\section{Analyse de la forme des pores}

La figure 3 représente les histogrammes de porosité des 5 niveaux analysés, les pores étant classés suivant leur indice d'allongement. Ces "spectres de forme" de la porosité nous permettent de définir 3 types morphologiques:

- les niveaux 20,65 et $80 p$ ont une porosité essentiellement tubulaire : $70 \%$ au moins de la surface porale sont constitués de pores ayant un in- dice d'allongement $<5,85 \%$ au moins sont constitués de pores ayant un indice d'allongement < 15 ;

- le niveau $80 f$ possède une porosité essentiellement fissurale : $88 \%$ de la surface porale sont constitués de pores ayant un indice d'allongement $>5$; les fissures très allongées (indice d'allongement $>75$ ) représentent encore $70 \%$ de la surface porale;

- le niveau 45 possède les 2 types de porosité : $56 \%$ de la surface porale est constituée de pores ayant un indice d'allongement $<5$; la proportion macropores arrondis/macropores allongés est donc mieux équilibrée dans ce niveau que dans les autres niveaux. On constate par ailleurs que les fissures de ce niveau sont beaucoup moins allongées que dans le niveau $80 f$ : l'indice d'allongement ne dépasse pas 55 .

Associés aux spectres de taille de la figure 2, ces spectres de forme nous permettent de décrire la morphologie porale des 5 zones d'étude : les petits pores tubulaires caractérisent le niveau 20 , les gros pores tubulaires caractérisent les niveaux 45,65 et $80 p$, les petites fissures peu allongées caractérisent le niveau 45 et les grosses fissures très allongées caractérisent le niveau $80 f$.

On constate ainsi une similitude morphologique entre les niveaux 65 (base de l'horizon polyédrique) et $80 p$ (cœur des prismes dans l'horizon 


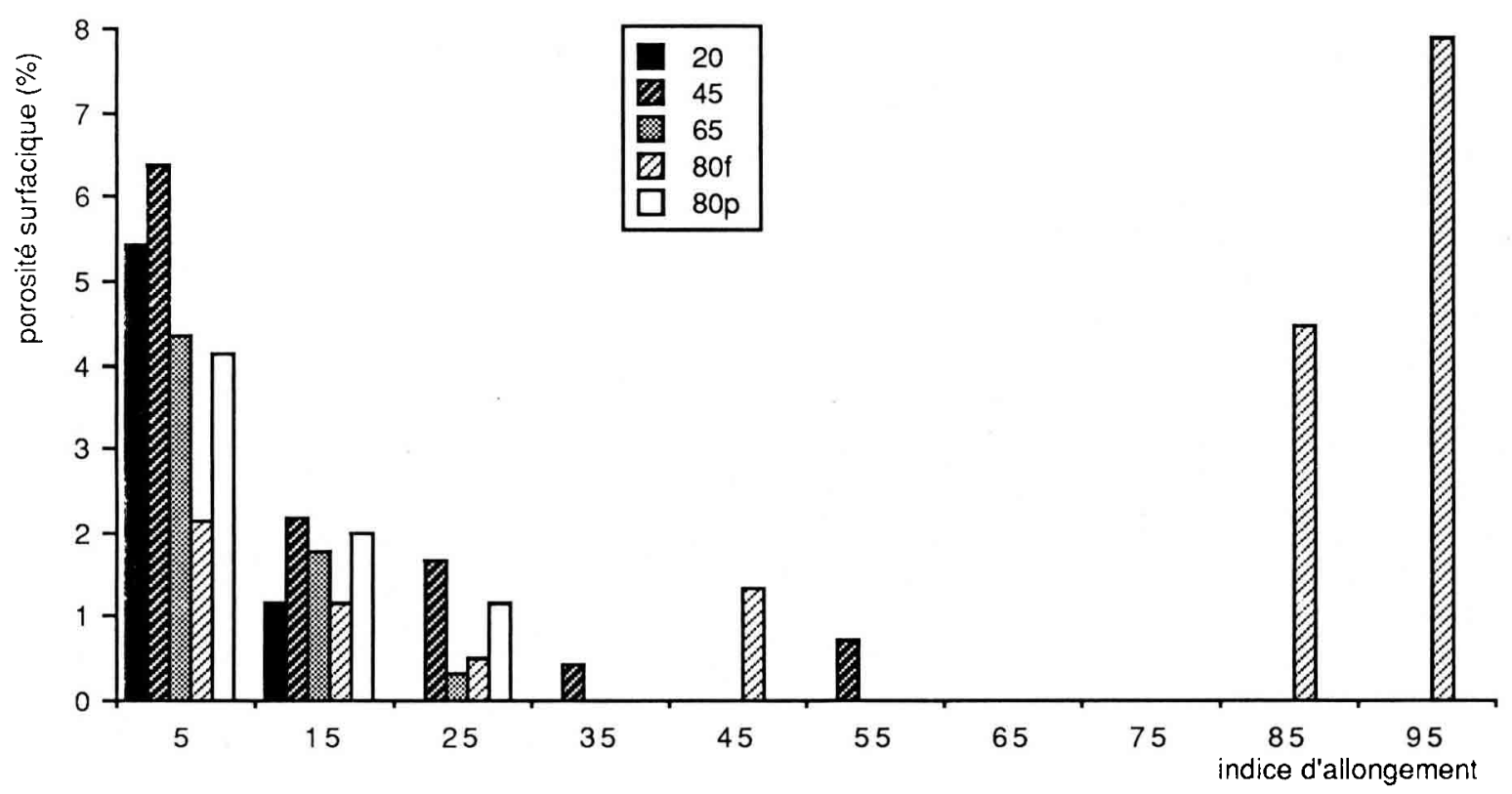

Fig 3. Histogrammes de la forme des pores pour les 5 niveaux analysés. Les classes de forme sont définies suivant l'indice d'allongement des pores.

prismatique), alors que les autres niveaux présentent des différences soit de taille, soit de forme des macropores.

\section{Analyse de l'orientation des pores}

L'indice d'orientation défini plus haut concerne principalement les pores allongés, qui représentent entre $18 \%$ (niveau 20 ) et $88 \%$ (niveau 80 ) de la macroporosité surfacique. L'histogramme de la figure 4 représente la répartition des pores selon leur orientation, par classe d'angles de $10^{\circ}$. Le niveau $80 f$ se démarque nettement des autres zones, avec une orientation préférentielle très marquée : $76 \%$ de la macroporosité surfacique est constituée de pores dont l'indice d'orientation est compris entre $80^{\circ}$ et $100^{\circ}$; les coupes analysées étant verticales, cet horizon est donc caractérisé par la présence de fissures essen-

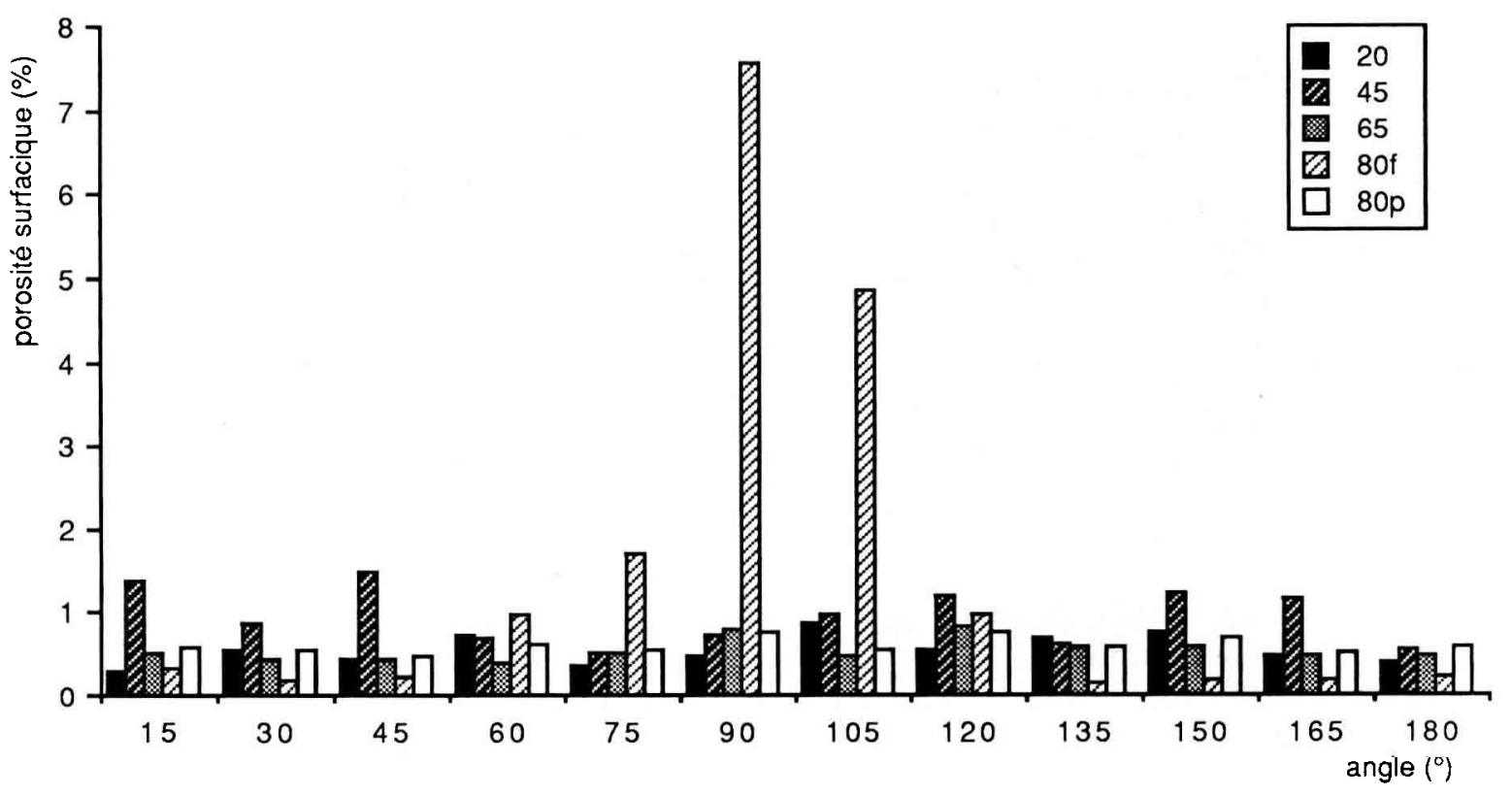

Fig 4. Histogrammes de l'orientation des pores pour les 5 niveaux analysés. Les classes d'angle sont définies suivant l'indice d'orientation des pores. 
tiellement verticales, lui conférant une anisotropie importante. Les 4 autres niveaux ne présentent pas d'orientation préférentielle : les histogrammes plats dénotent une isotropie de la macroporosité. Cela est peu significatif pour les niveaux 20,65 et 80 p où les pores sont essentiellement arrondis; en revanche ce résultat est important pour le niveau 45 qui possède de nombreuses fissures : contrairement aux fissures de I'horizon prismatique sous-jacent, les fissures de I'horizon polyédrique ne présentent pas d'orientation préférentielle. Le paramètre d'orientation retenu est donc un complément utile des paramètres de taille et de forme, en renseignant sur une éventuelle anisotropie de la porosité fissurale.

\section{Bilan de porosité et conséquences agronomiques}

La figure 5 propose une représentation schématique de la macroporosité au sein du profil. Les pores ont ici été classés en 2 catégories (pores ronds et fissures), la distinction entre ces 2 types de pores étant établie en attribuant à l'indice d'allongement un seuil de 5 . Cette simplification par rapport à l'analyse précédente ne permettra donc pas une analyse morphologique fine, mais nous servira à proposer un schéma pour le fonctionnement agronomique de ce sol sous verger.

Dans l'horizon supérieur, la macroporosité surfacique est faible $(6,5 \%)$. Ces résultat, fréquent pour les horizons de surface de texture limono-sableuse, confirme le compactage consta- té lors de la prospection pédologique (densité apparente de 1,73). Notre analyse morphologique montre que la macroporosité est constituée de très nombreux pores tubulaires qui, tout en faisant partie de la macroporosité, sont de petite taille : leur diamètre équivalent moyen est d'environ 250 $\mu \mathrm{m}$; bien que compacté, cet horizon ne constitue donc pas d'obstacle à la pénétration racinaire.

L'horizon polyédrique sous-jacent a une macroporosité surfacique beaucoup plus élevée ( $11,4 \%$ pour le niveau 45$)$, ce qui confirme là encore le résultat obtenu lors de la prospection pédologique (densité apparente de 1,46); morphologiquement, on a pu montrer que cette porosité est constituée de pores tubulaires, sensiblement plus gros qu'en surface, et d'un réseau isotrope de fines fissures. Cet horizon pourrait donc constituer un milieu favorable tant à la pénétration racinaire qu'aux conditions de circulation de l'eau. Cependant, notre analyse a pu mettre en évidence, à la base de cet horizon, un niveau beaucoup plus compacté; ce compactage se traduit pas une réduction du nombre des pores tubulaires, sans modification notable de leur taille, et surtout par une diminution de plus de moitié de la porosité fissurale. Ce niveau constitue donc un frein à l'écoulement vertical de l'eau dans le sol, ce qui peut provoquer les phénomènes d'hydromorphie constatés dans tout cet horizon et nuire à sa colonisation racinaire.

Dans I'horizon inférieur prismatique, on a pu mettre en évidence une macroporosité bimodale, constituée de grandes fissures verticales isolant des prismes compactés, où la porosité est identi-

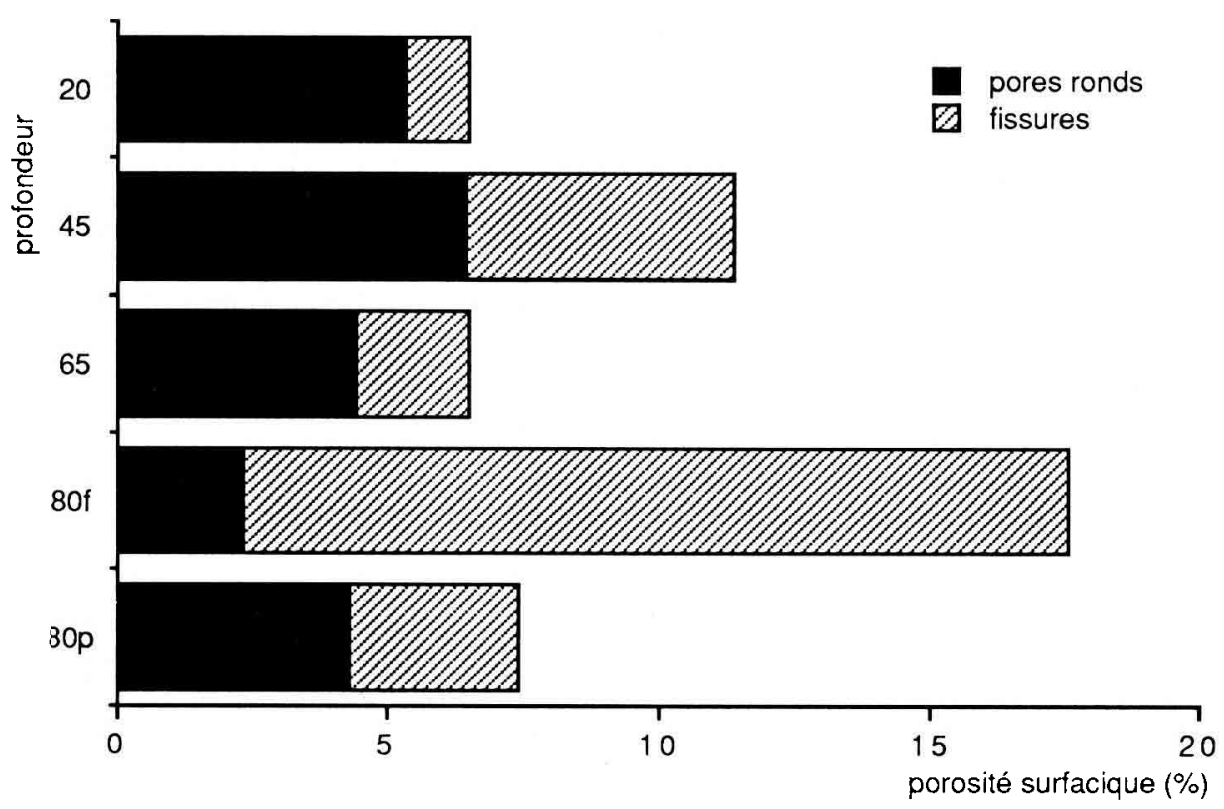

Fig 5. Profil de porosité surfacique des 5 niveaux analysés, après regroupement des pores en 2 catégories : pores ronds (indice d'allongement $<5$ ) et fissures. 
que (morphologiquement et quantitativement) à celle du niveau 65; la taille et l'orientation des fissures peuvent assurer des conditions d'écoulement de l'eau favorables dans cet horizon. La mesure de densité apparente effectuée lors de la prospection pédologique $(1,60)$ ne rend pas compte du caractère bimodal de la macroporosité dans cet horizon.

\section{CONCLUSION}

Là où la description pédologique apporte une caractérisation qualitative de la structure des horizons, et là où les mesures densimétriques permettent une quantification globale de la porosité, l'analyse d'image permet de décrire avec précision l'évolution de la macroporosité au sein du profil. Les principaux apports de la méthode utilisée tiennent d'une part à une caractérisation individuelle des pores, et non plus globale de la porosité, et d'autre part à une description morphologique, permettant la prise en compte de critères aussi essentiels que la forme et l'orientation des pores : les conditions de circulation d'eau dans le sol et la colonisation racinaire sont étroitement dépendantes de ces paramètres.

Les limites de notre approche tiennent surtout au choix de l'échelle d'étude : la taille du pixel détermine la taille du champ analysé, ce qui peut être contraignant lorsque les pores sont très espacés, comme ce fut le cas pour les fissures de I'horizon prismatique. Malgré ses apports, la méthode proposée ici ne saurait donc se substituter aux méthodes physiques de mesure de la porosité, qui présentent l'avantage d'être indépendantes de l'échelle d'analyse, et qui permettent une estimation volumique de la porosité. Une telle description morphologique s'avère donc utile pour donner des détails sur la distribution en taille et en forme de la porosité d'un sol à une échelle donnée, mais l'analyse de l'ensemble du spectre poral nécessite l'utilisation d'autres techniques, comme la porosimétrie à mercure pour la porosité fine. Loin de s'opposer, les approches "physiques" et "morphologiques» paraissent donc complémentaires pour appréhender la porosité d'un sol dans sa globabilité, quels que soient le type de sol et son contexte agronomique.

\section{RÉFÉRENCES}

Bouma J, Jongerius A, Boersma O, Jager A, Schoonderbeek $D$ (1977) The function of different types of macropores during saturated flow through four swelling soil horizons. Soil Sci Soc Am J 41, 945950

Ben Rhaïem H, Pons CH, Tessier D (1987) Factors affecting the microstructure of smectites: role of cation and history of applied stresses. In: Proc Int Clay Conf (LG Schultz, H van Olphen, FA Mumpten, eds) The Clay miner Soc Bloomington, Indiana, 292-297

Bui EN, Mermut AR (1989) Orientation of planar voids in vertisols and soils with vertic properties. Soil Sci Soc Am J 53, 171-178

Bui EN, Mermut AR, Santos MCD (1989) Microscopic and ultramicroscopic porosity of an oxisol as determined by image analysis and water retention. Soil Sci Soc Am J 53, 661-665

Bullock P, Thomasson AJ (1979) Rothamsted studies of soil structure. II. Measurement and characterisation of macroporosity by image analysis and comparison with data from water retention measurements. J Soil Sci 30, 391-413

Coster M, Chermant JL (1985) Précis d'analyse d'image. CNRS, Paris

Croney D, Coleman JD (1954) Soil structure in relation to soil suction (pF). J Soil Sci 5, 75-84

Daniel T, Gapais D, Labit C, Le Corre C (1987) An application of textural and morphological image processing to deformation analysis within granitic rocks. Acta Stereol 6/3, 1041-1046

Douglas JT (1986) Macroporosity and permeability of some soil cores from England and France. Goederma 37, 221-231

Edwards WM, Norton LD, Redmond CE (1988) Characterizing macrospores that affect infiltration into nontilled soil. Soil Sci Soc Am J 52, 483-487

Germann P, Beven K (1981) Water flow in soil macropores. I. An experimental approach. J Soil Sci 32, $1-13$

Hallaire V (1988) La fissuration d'un sol argileux au cours du dessèchement. II. Modélisation morphologique. Agronomie 8, 273-280

Hallaire V (1989) A 3-dimensional description of cracks networks in clayey soils, using image analysis and a structural model. Acta Stereol 8/2, 301306

Jongerius A, Schoonderbeek D, Jager A, Kowalinski $S$ (1972) Electro-optical soil porosity investigation by means of Quantimet-B equipment. Geoderma 7. 177-198

Launeau P, Bouchez JL, Benn K (1990) Shape prefered orientation of object population: automatic analysis of digitized images. Technophysics 180, 201211

Lawrence GP, Payne D, Greenland DJ (1979) Pore size distribution in critical point and freeze dried aggregates from clay subsoils. J Soil Sci 30, 499-516

Lorgeoux J (1981) Cartographie détaillée des sols du domaine expérimental du Centre Technique Inter- 
professionnel des Fruits et des Légumes de StEpain (Indre-et-Loire). SDS 246 ENSAR-INRA, Rennes, France

Low AJ, Douglas LA, Platt DW (1982) Soil pore orientation and faults. Soil Sci Soc Am J 46, 789-792

Mackie-Dawson LA, Mullins CE, Kirkland JA, Fitzpatrick EA (1988) The determination of the macroporosity of impregnated blocks of a clay soil and its relation to volumetric water content. J Soil Sci 39 , 65-70

Murphy CP, Bullock P, Turner RH (1977) The measurement and characterisation of voids in soil thin sections by image analysis. Part I. Principles and techniques. J Soil Sci 28, 498-508

Newman ACD, Thomasson AJ (1979) Rothamsted studies of soil structure. III. Pore size distribution and shrinkage processes. J Soil Sci 30, 415-439

Noesis (1991) A tutorial image processing. Noesis, Vélizy, France

Pagliai M, La Marca M, Lucamante G (1983) Micromorphometric and micromorphological investigations of a clay loam soil in viticulture under zero and conventional tillage. J Soil Sci 34, 391-403
Reeve MJ, Hall DGM (1978) Shrinkage in clayey subsoils of contrasting structure. J Soil Sci 29, 315-323

Ringrose-Voase AJ (1987) A scheme for the quantitative descripton of soil macrostructure by image analysis. J Soil Sci 38, 343-356

Scott GJT, Webster R, Nortcliff S (1988) The topology of pore structure in cracking clay soil. I. The estimation of numerical density. J Soil Sci 37, 303-314

Serra J (1982) Image analysis and mathematical morphology. Acad Press, Londres

Smettem KRJ, Collis-George N (1985) The influence of cylindrical macropores on steady-state infiltration in a soil under pasture. $J$ Hydrol 79, 107-114

Sullivan LA, Koppi AJ (1987) Multi-orientation thin sectioning for the determination of 3-D morphology. $J$ Soil Sci 38, 383-385

Tessier D (1984) Étude expérimentale de l'organisation des matériaux argileux. Thèse Univ Paris, Paris, France. INRA Publications Versailles

Warner GS, Nieber JL, Moore ID, Geise RA (1989) Characterizing macropores in soil by computed tomography. Soil Sci Soc Am J 53, 653-660 